\title{
References
}

1. Fleishier, G. A., C. S. Potter and K. G. Whkim, Proc. Soc. exp. Biol. Med., 103, 229 (1960). - 2. Fleisher, G. A. and K. G. Wakim, Proc. Soc. exp. Biol. Med. 106, 283 (1961). - 3. Rosenthat, O., S. K. Thind and N. Conger, Abstr. Amer. chem. Soc., 138 th Meeting, New York, 10C, (1960). - 4. Borst, P. and E. M. Peeters, Biochim. biophysica Acta (Amsterdam) 54, 188 (1961). -5. BoYd, J. W., Biochem. J., 81, 434 (1961). - 6. BoYDE, T. R. C. and A. L. LATNER, Biochem. J., 82, 51P (1962). - 7. BoYDE, T. R.C. in preparation. - 8. Martinez-Carrion, M., F. Riva, C. Turano and P. Faselia, Biochem. Biophys. Res. Comm. 20, 206 (1965). - 9. Martinez-Carrion, M., C. Turano, M. Chiancore, F. Bossa, A. Giartosio, F. Riva and P. Fasella, J. biol. Chemistry 242, 2397 (1967). - 10. Martinez-Carrion, M. and D. Tiemier, Biochemistry (USA) 6, 1715 (1967). - 11. Boyde, T. R. C., Biochem. J., 106, 581 (1968). - 12. Boyde, T. R. C., M. D. Thesrs, University of London, 1967. - 13. Boxde, T. R. C. and I. F. PrYMe, Clin. Chim. Acta (Amsterdam) 21, 9 (1968). -
14. Karmen, A., J. Clin. Invest., 34, 131 (1955). - 15. Ideo, G., P. M. Mannucci, E. MunN, G. Funelir and N. Dioguardi, Boll. Soc. ital. biol. sper., 41, 942, 954 (1965). - 16. Massarat, S. and N. LaNG, Klin. Wschr., 43,602 (1965). - 17. Schwartz, M. K. and O. Bodansky, Amer. J. Med.40,231 (1966).-18. VilLA, L., N. Dioguardi and A.Agoston, Klin. Wschr. 45, 44 (1967). - 19. Neumieister, E., P. Otro, E. Schmidt and F. W. Schmidt, Med. Welt, 1497 (1965). - 20. Romer, W. C. and S. J. La Mancusa, Clin. Chem. (New York) 11, 131 (1965). - 21. FleIsher, G. A. and K. G. WAKIM, J. Laborat. clin. Med. (S. Louis) 61, 98 (1963). - 22. BoxDE, T. R. C., Enzymol. biol. clin., in press. - 23. Notarbartolo, A., F. Giglio, S. LeMoli, G. Mrgneco and L. Pagliaro, Boll. Soc. ital. biol. sper., 42, 328 (1966). - 24. RotzsCH, W. and K. W. Wenzer, Acta biol. med. german., 17, 561 (1966). 25. Retrman, S. and S. Frankel, Amer. J. clin. Path., 28,56 (1957).
T. R. C. Boyde, M. D.

Dept. Clin. Biochem.

Royal Victoria Infirmary

Newcastle upon Tyne/England

\section{Untersuchungen über das Schicksal von Dextran im Organismus von Meerschweinchen}

\author{
Vón K. H. Ebert, G. Schenk ${ }^{1}$ ) und R. Scholz ${ }^{2}$ ) \\ Institut für Technische Chemie der Technischen Hocbscbule München
}

(Eingegangen am 7. Mai 1968)

Nach einmaliger Injektion von tritiierten Dextranfraktionen mit unterschiedlichen, genau definierten Molekulargewichten, wurde an Meerschweinchen die Verweildauer des Dextrans im Blut, seine Ausscheidung, Metabolisierung und Retention in den Geweben untersucht. Nahezu $100 \%$ der injizierten Tritiumaktivitäten konnten im Blut, in den verschiedenen Organen und in den Ausscheidungsprodukten wiedergefunden werden. Die Verweildauer im Blut und die Ausscheidung über Nieren und Darm zeigen eine hohe Abhängigkeit vom Molekulargewicht des injizierten Dextrans. Eine Ausscheidungsschwelle scheint in den Nieren für Molekulargewichte über $50000 \mathrm{zu}$ bestehen. Die Bildung von Tritiumwasser bestätigt, daß die Dextrane abgebaut und in den intermediären Stoffwechsel eingeschleust werden. Diese Abbaureaktionen zeigen eine molekulargewichtsabhängige Induktionsperiode von ein bis drei Tagen. Niedermolekulare Fraktionen werden bedeutend schneller umgesetzt als höhermolekulare. Dextran wird in den meisten Organen retiniert, wobei hinsichtlich des Ausmaßes dieser Retention folgende Reihenfolge aufgestellt werden kann: Milz, Haut, Lunge, Leber, Niere, Fettgewebe, Skelettmuskulatur. Im Gehirn sind nur Spuren nachweisbar. Mit Ausnahme der Milz ist in den meisten Organen die Dextranretention nur angedeutet molekulargewichtsabhängig. Die Verweildauer der Dextrane im Blut wird vorwiegend von der Ausscheidung über die Nieren bestimmt, Metabolisierung oder Retention in den Geweben haben dagegen keinen wesentlichen Einfluß. Das Verhalten der Milz zeigt jedoch, daß dem reticuloendothelialen System eine bedeutende Rolle bei der Reinigung des Blutes von höhermolekularen Dextranen zukommt.

\section{Studies on the fate of injected dextran in the guinea pig}

Guinea pigs were given single injections of tritiated dextran fractions of different and precisely known molecular weights. The persistence of dextran in the blood, its excretion, metabolism and retention in the tissues were studied. Practically $100 \%$ of the injected tritium activity was found in the blood, various organs and excretory products. The persistence of the dextran in the blood and its excretion via the kidneys and intestine showed a marked dependence on the molecular weight. There appears to be an excretory threshold in the kidneys for molecular weights above 50000. The appearance of tritium water showed that the dextrans are degraded and thereby become involved in intermediary metabolism. These degradation reactions are subject to an induction period of one to three days, depending on the molecular weight of the dextran. Low molecular weight fractions are degraded significantly more rapidly than high molecular weight fractions. Dextran is retained by most organs to an extent represented by the following order: spleen, skin, lungs, liver, kidneys, adipose tissue, skeletal muscle, and only a trace in the brain. With the exception of the spleen, the retention of dextran in most organs is only slightly affected by molecular weight. The persistence of dextrans in the blood is determined chiefly by the excretion via the kidneys, and the metabolism or retention in the tissues have essentially no effect. The behaviour of the spleen shows, however, that the reticuloendothelial system plays a significant part in the purification of the blood by the removal of high molecular weight dextrans.

1) derzeitige Adresse: Knoll AG, Chemische Fabriken, 67 Ludwigshafen a. Rh.

2) z. Z. Johnson Research Foundation, School of Medicine, University of Pennsylvania, Philadelphia/USA. 
Seit mehr als zwei Jahrzehnten werden partiell abgebaute Dextrane als Blutplasma-Ersatzmittel verwendet. Untersuchungen über das Schicksal von Dextran im Organismus haben u. a. ergeben, daß seine Verweildauer im Blut und seine Ausscheidung in den Urin molekulargewichtsabhängig sind, wobei niedermolekulare Anteile den Organismus schneller verlassen (1-6). In den Kot erfolgt ebenfalls eine Ausscheidung $(7,8)$. In einigen Organen wird Dextran zurückgehalten, was aufgrund der längeren Nachweisbarkeit im Gewebe im Vergleich zum Blut gefolgert wurde (9). Versuche mit ${ }^{14} \mathrm{C}$-markiertem Dextran zeigten darüber hinaus, daß Dextran teilweise metabolisiert wird, da in der Atemluft der Versuchstiere radioaktives $\mathrm{CO}_{2}(7,9)$ und im Urin radioaktive Glucose (10) gefunden wurden. Außerdem konnten auch ${ }^{14} \mathrm{C}$-A ktivitäten in körpereigenen Substanzen nachgewiesen werden (11). Die Abbaugeschwindigkeit des Dextrans ist jedoch relativ langsam. An; seinem Abbau sollen mehrere Enzyme beteiligt sein (12).

Die meisten experimentellen Ergebnisse wurden bei der Aufklärung von Einzelproblemen erhalten und haben oft nur qualitativen Charakter. Sie können nicht ohne weiteres miteinander verglichen werden, weil die Experimente mit Dextranen verschiedener Herkunft oder ungenügender Spezifikation durchgeführt wurden. Quantitative Untersuchungen sind u. W. nur von TERrY und Mitarbeitern (9) und HellmanN (7) beschrieben worden, die Bilanzen nach Verabreichung von ${ }^{14} \mathrm{C}$-markiertem Dextran $\left(\overline{\mathrm{M}}_{\mathrm{w}}=66000 ; \overline{\mathrm{M}}_{\mathrm{n}}=\right.$ 30200) $)^{3}$ aufgestellt haben. In Versuchen an Hunden konnten nach 13 Tagen 95\% der verabreichten Dextranmenge im Urin, in Atemluft und Körper wiedergefunden werden (9). In klinischen Versuchen wurde die Ausscheidung der Radioaktivität verfolgt und dabei in 10 Tagen $64 \%$ der injizierten Aktivität im Urin, $26 \%$ im $\mathrm{CO}_{2}$ der Atemluft und $2 \%$ im Kot nachgewiesen.

Das Ziel unserer Untersuchungen war, weitere quantitative Angaben über das Schicksal von Dextranen im Organismus in Abhängigkeit vom Molekulargewicht $\mathrm{zu}$ erhalten. Wir verwendeten Meerschweinchen als Versuchstiere und fünf exakt definierte Fraktionen eines tritiummarkierten Dextrans.

\section{Methodik}

1. Herstellung von tritiummarkiertem, particll abgebautem Dextran.

Saccharose wurde nach der Methode von WILZBACH radioaktiv markiert (13) und mit dem Enzym Dextransaccharase aus Leuconostoc mesenteroides B 512F zu nativem Dextran umgesetzt. Infolge der absoluten Spezifität dieses Enzyms (14) werden keine radioaktiven Radiolyseprodukte zu hochmolekularen Substanzen aufgebaut. Durch mehrtägige Dialyse und zweimaliges Umfällen mit Äthanol wurde das native Dextran von niedermolekularen Produkten befreit und anschließend durch Ultraschallbehandlung partiell abgebaut. Das so erhaltene ${ }^{3} \mathrm{~F}[-\mathrm{Dextran}$ wurde durch Gelfraktionierung in bezug auf seine Molekulargewichtsver-

3) $\bar{M}_{n}=$ Zahlenmittelwert des Molekulargewichts, $\bar{M}_{w}=$ Gewichtsmittelwerte des Molekulargewichts. teilung charakterisiert (15), wobei sich ein $\overline{\mathrm{M}}_{\mathbf{n}}$-Wert von 30700 und $\overline{\mathbf{M}}_{\mathrm{w}}$-Wert von 118900 errechnen ließ. Mit der Endgruppenbestimmung nach SomoGYI wurde ein $\overline{\mathbf{M}}_{n}$-Wert von 24400 gefunden. Die spezifische Aktivität betrug 6,7 $10^{5} \mathrm{Imp}$./Min./mg Dextran.

\section{Dextran-Fraktionierung}

Nach der Molekulargewichtsverteilung überstrich das ${ }^{3} \mathrm{H}-D e x t r a n$ den Bereich, der in unseren Untersuchungen exfaßt werden sollte. Zur Herstellung der Injektionslösungen wurden Dextrane der Firma Pharmacia AB (Dextran 500, 150 und 40) im Verhältnis 1:2:3 mit ${ }^{3} \mathrm{H}-$ Dextran vermischt und einer Fällfraktionierung unterworfen (16). Dabei wuirde aus der wäßr. Dextranlösung durch langsame Zugabe von Äthanol das Polymere ausgefällt. Wir erhielten fünf Fraktionen, deren Molekulargewichtsver-

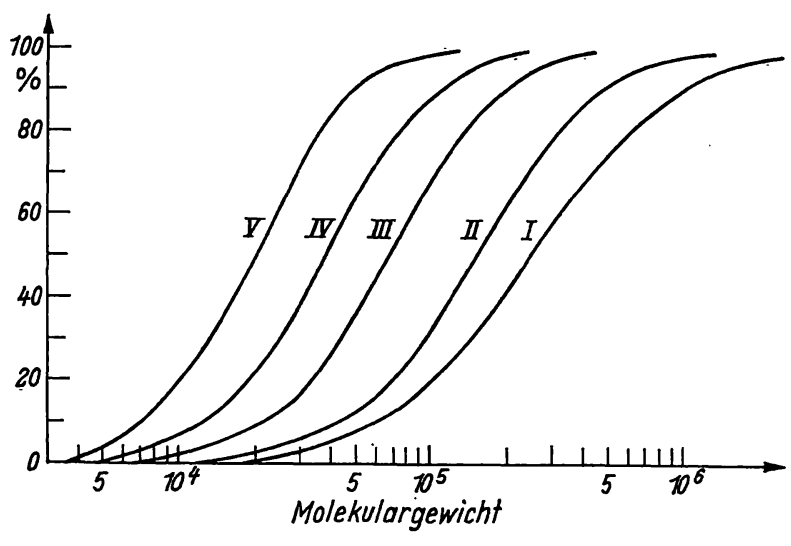

Abb. 1

Molekulargewichtsverteilung der Dextranfraktionen $\mathrm{I}-\mathrm{V}$ bestimmt durch Gelfraktionierung

teilung durch Gelfraktionierung (Abb. 1) und deren $\overline{\mathbf{M}}_{\mathbf{n}}$-Werte zusätzlich durch Endgruppenanalyse bestimmt wurden. Die spezifischen Aktivitäten der einzelnen Fraktionen wurden im Flüssigkeitsszintillationszähler vermessen. In Tabelle 1 sind die Daten, die diese fünf Fraktionen charakterisieren, zusammengefaßt. Die Injektionslösungen enthielten jeweils $8 \mathrm{~g}$ Dextran und $0,9 \mathrm{~g} \mathrm{NaCl}$ in $100 \mathrm{ml}$.

Tab. 1

Molekulargewichte und spezifische Aktivitäten der Dextranfraktionén $\mathrm{I}-\mathrm{V}$

\begin{tabular}{ccccc}
\hline $\begin{array}{c}\text { Dextran- } \\
\text { fraktion } \\
\text { Nr. }\end{array}$ & $\begin{array}{c}\bar{M}_{\mathbf{n}} \\
\text { (End- } \\
\text { gruppen- } \\
\text { analyse) }\end{array}$ & $\begin{array}{c}\bar{M}_{\mathbf{n}} \\
\text { (berechnet aus Gelfrak- } \\
\text { tionierung) }\end{array}$ & $\begin{array}{c}\bar{M}_{\text {Spez. Akti- }} \\
\text { vität } \\
\text { Imp./Min./mg } \\
\text { Dextran }\end{array}$ \\
\hline I & 134300 & 132600 & 440300 & 17967 \\
II & 87400 & 96600 & 225000 & 18184 \\
III & 45500 & 45500 & 94800 & 16044 \\
IV & 20300 & 26900 & 52400 & 12541 \\
V & 10000 & 15200 & 25800 & 8874 \\
\hline
\end{tabular}

3. Tiermaterial und Durchführung der Versuche

Wir verwendeten weibliche Albinomeerschweinchen, Stamm Pirbright (Tierzüchterei P. Bäumler, 819 Wolfratshausen) im Gewicht $z$ wischen 200 und $330 \mathrm{~g}$. Die Tiere erhielten eine kohlenhydratreiche Standarddiät („Hopefarms") und Wasser ad libitum. Die sterile Dextran-NaCl-Lösung wurde sehr langsam in die $V$ ena saphena injiziert, die durch einen kleinen Hautschnitt in oberflächlicher Âthernarkose freigelegt wurde. Die verabreichte Dextranmenge lag zwischen 1 und 2 g Substanz pro kg Körpergewicht.

In Experimenten, bei denen die Retention im Blut, in Leber, Nieren und Milz untersucht wurde, wurden die Tiere in Einzelkäfigen gehalten, deren Boden mit reichlich Sägemehl bedeckt war. Zur Vermeidung von stärkerer Verunreinigung des Fells durch radioaktive Urin- und Kotspuren wurden die Tiere mehrmals in saubere Käfige umgesetzt. 
Für Expcrimente, in denen zusätzlich die Ausscheidung von ${ }^{3} \mathrm{H}$-Aktivitäten verfolgt wurde, haben wir besondere, hermetisch abgeschlossene Stoffwechselkäfige konstruiert. Die Tiere saßen auf einem Kunststoffrost in einem Plexiglasrohr (innerer Durchmesser $8 \mathrm{~cm}$, Länge $20 \mathrm{~cm}$ ), dessen Stirnseiten mit konischen Kunststoffeinsätzen luftdicht verschlossen waren. An der Kopfseite befand sich das metallene Saugrohr einer Wasserflasche, eine Öfnung für die Einführung von Futterpellets und der Auslaß für die Atemluft. An der gegenüberliegenden Seite wurde trockene Frischluft aus einer Preßluftflasche eingeführt. Die Strömungsgeschwindigkeit von etwa $200 \mathrm{~m} / / \mathrm{Min}$. wurde mittels eines Rotameters kontrolliert. Die gesamte Abluft wurde über einen mit Silikagel beschickten Trockenturm geführt, in dem der ausgeschiedene Wasserdampf adsorbiert wurde. Am tiefsten Punkt des leicht geneigten Käfigs befand sich der Urinauslaß. Der Urin floß durch eine Schlauchverbindung in ein dicht verschlossenes Auffanggefäß, dessen Luftraum wiederum mit dem Käfig verbunden war, um einen raschen $A b f l u ß$ zu gewährleisten. Diese Gefäße wurden sofort ausgewechselt, sobald eine Urinentleerung beobachtet wurde und in der Kälte aufbewahrt. Der Kot wurde durch ein Sieb zurückgehalten und täglich zweimal gesammelt, wobei die Rückseite des Käfigs geöffnet werden mußte. Anschließend wurden jeweils Käfigboden und Rost gründlich mit 5proz. RBS-Lösung (Detergens und Desinfiziens, Firma Carl Roth, Karlsruhe) und mit Wasser abgespritzt, um die ausgeschiedenen ${ }^{3} \mathrm{H}$-Aktivitäten quantitativ $\mathrm{zu}$ erfassen und um eine bakterielle Besiedlung des Käfigs zu unterdrücken.

Die Tiere wurden 3 bzw. 6 Tage nach der Dextraninjektion aus den Käfigen genommen und durch Schädelschlag betäubt. Aus den eröffneten Halsschlagadern wurde Blut zur Analyse aufgefangen, dem zur Gerinnungshemmung Heparin („Liquemin" Roche) zugesetzt wurde. Die weitere Entblutung erfolgte nach Dekapitation. Nach Zerlegung des Tierkörpers wurden aus den einzelnen Organen jeweils 3 bis 8 Gewebsproben (100-200 mg Frischgewicht; bis zu 50 Proben je Tier) entnommen und sofort gewogen. Dabei sollte ein möglichst repräsentativer Querschnitt durch sämtliche Organbezirke erfaßt werden, z. B. periphere und zentrale Anteile mehrerer Leberlappen, Skelettmuskulatur im Rumpf- und Extremitätenbereich, Haut und Unterhautfettgewebe aus verschiedenen Körperregionen, verschiedene Darmabschnitte usw. Diese verschiedenen Organbezirke wurden in Tabelle 2 und 3 jedoch nur dann gesondert aufgeführt, wenn dcutliche Unterschiede in den Ergebnissen zu erkennen waren. Im übrigen wurden Mittelwerte aus sämtlichen Proben eines Organs angegeben.

\section{Aufarbeitung der Proben und Aktivitätsmessung}

Die einzelnen Gewebsproben wurden gefriergetrocknet und anschließend das Trockengewicht bestimmt. Tabelle 2 enthält Mittelwcrte des Trockengewichtsanteiles der Organfrischgewichtc. Der Kot wurde nach der Gefriertrocknung in einer Reibschale homogenisiert.

Die Tritiumaktivitäten in den wasserfreien Proben wurden nach der Methode von KALBERER und RutschmanN (17) analysiert, bei der die einzelne Probe im Sauerstoffstrom verbrannt und die Aktivität des entstandenen Tritiumwassers im Flüssigkeitsszintillationszähler gemessen wird. Die Tritiumaktivität im Wasser der Atemluft, das aus dem Silikagel desorbiert wurde, sowie im Abkondensat des Urins, erhielten wir durch dirckte Messung im Flüssigkeitsszintillationszähler.

\section{Ergebnisse und Diskussion}

\section{Verweildauer im Blut}

Wie oben erwähnt, sind Dextrane verschiedenen Molekulargewichts nach intravenöser Injektion unterschiedlich lange im Blut nachweisbar. In unseren Versuchen haben wir Meerschweinchen verschiedene tritiummarkierte Dextranfraktionen injiziert, deren mittlere Molekulargewichte den Bereich zwischen 15000 und 150000 umfaßten. Der Anteil der injizierten Tritium-Aktivität, der nach 3 und 6 Tagen im Blut nachweisbar war, ist in Abbildung 2 gegen das mittlere Molekulargewicht dieser Fraktionen aufgetragen. Wir haben bei dieser Darstellung das Zahlenmittel des Molekulargewichts $\left(\overline{\mathbf{M}}_{n}\right)$ als Vergleichswert gewählt. Die Gewichtsmittelwerte $\left(\overline{\mathrm{M}}_{\mathrm{w}}\right)$ der einzelnen Fraktionen sind in Tabelle 1 mit angegeben. Drei Tage nach der Injektion besteht eine angenähert lineare Beziehung zwischen dem noch im Blut enthaltenen Anteil der injizierten ${ }^{3} \mathrm{H}$-Aktivität und dem Logarithmus von $\overline{\mathbf{M}}_{n}$. Von den hochmolekularen Fraktionen sind zu diesem Zeitpunkt noch $30-40 \%$, von den niedermolekularen Fraktionen dagegen weniger als 10\% nachweisbar. Das Ergebnis nach sechstägiger Verweildauer zeigt, daß eine Line-

Tab. 2

Tritium-Aktivität in verschiedenen Organen

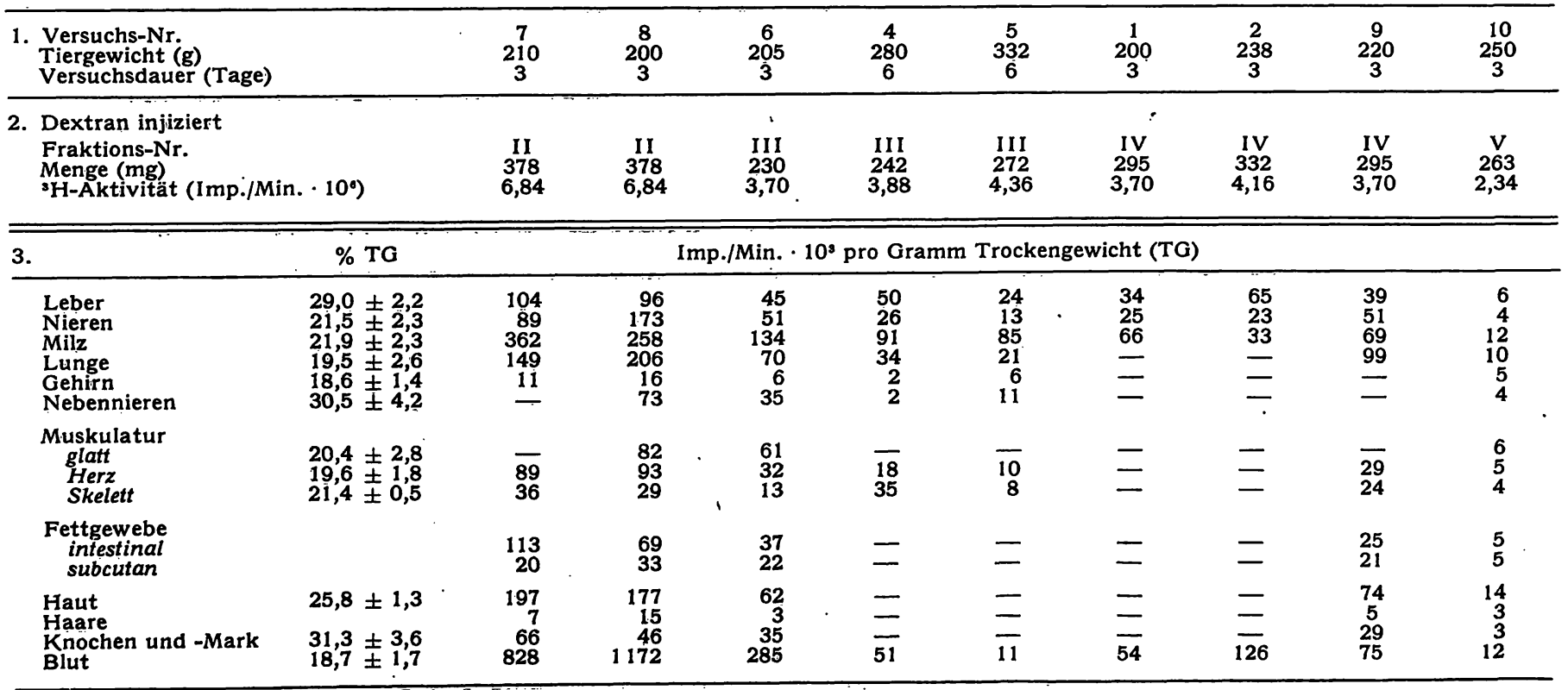


Tab. 3

Bilanzen der Tritium-Aktivitäten in Prozent der injizierten Menge

\begin{tabular}{|c|c|c|c|c|c|c|c|c|c|}
\hline $\begin{array}{l}\text { 1. Versuchs-Nr. } \\
\text { Tiergewicht (g) } \\
\text { Versuchsdauer (Tage) }\end{array}$ & ${ }_{3}^{7}$ & $\begin{array}{c}800 \\
3\end{array}$ & $\begin{array}{c}6 \\
205 \\
3\end{array}$ & $\begin{array}{c}4 \\
280 \\
6\end{array}$ & $\begin{array}{c}5 \\
232 \\
6\end{array}$ & 200 & $\begin{array}{c}238 \\
3\end{array}$ & $\begin{array}{c}9 \\
220 \\
3\end{array}$ & $\begin{array}{c}10 \\
250 \\
3\end{array}$ \\
\hline $\begin{array}{l}\text { 2. Dextran injiziert } \\
\text { Fraktions-Nr. } \\
\text { Menge (mg) } \\
\left.\text { 'H-Aktivität (Imp./Min. } \cdot 10^{\circ}\right)\end{array}$ & $\begin{array}{l}11 \\
378 \\
6,84\end{array}$ & $\begin{array}{l}11 \\
378 \\
6,84\end{array}$ & $\begin{array}{l}\text { III } \\
230 \\
3,70\end{array}$ & $\begin{array}{l}\text { III } \\
242 \\
3,88\end{array}$ & $\begin{array}{l}111 \\
272 \\
4,36\end{array}$ & $\begin{array}{l}\text { IV } \\
295 \\
3,70\end{array}$ & $\begin{array}{l}\text { IV } \\
332 \\
4,16\end{array}$ & $\begin{array}{l}\text { IV } \\
295 \\
3,70\end{array}$ & $\begin{array}{c}V \\
263 \\
2,34\end{array}$ \\
\hline
\end{tabular}

3. 'H-Aktivität im Körper (\%)
1. Leber
2. Nieren
3. Milz
5. Lunge
6. Muskulatur
7. Fettgewebe
8. Haut
9. Knochen und -Mark
10. Darm
Summe $1-10$
11. Blut
12. Körperwasser
Summe $1-12$

\begin{tabular}{|c|c|c|c|c|c|c|c|c|}
\hline $\begin{array}{r}3,2 \\
0,7 \\
0,4 \\
0,7 \\
0,1 \\
10,3 \\
1,8 \\
11,8 \\
0,8 \\
0,7\end{array}$ & $\begin{array}{r}3,1 \\
1,1 \\
0,2 \\
1,1 \\
0,1 \\
6,1 \\
1,8 \\
10,8 \\
0,8 \\
0,7 \\
\end{array}$ & $\begin{array}{l}3,0 \\
0,7 \\
0,2 \\
0,5 \\
0,1 \\
6,5 \\
1,4 \\
9,2 \\
0,8 \\
0,5\end{array}$ & $\begin{array}{l}3,7 \\
0,4 \\
0,1 \\
0,5 \\
\widetilde{9}, 0 \\
2,0 \\
\mathbb{8}, 0 * \\
0,8 * \\
0,4 *\end{array}$ & $\begin{array}{l}2,0 \\
0,2 \\
0,1 \\
0,4 \\
0,1 \\
7,6 \\
1,4 \\
8,0 * \\
0,8^{*} \\
0,4^{*}\end{array}$ & $\begin{array}{l}2,4 \\
0,4 \\
0,1 \\
1,0 * \\
7,0 * \\
1,5 * \\
8,0^{*} \\
0,8^{*} \\
0,4^{*}\end{array}$ & $\begin{array}{l}5,8 \\
0,3 \\
0,8 \\
1,0 * \\
7,0 * \\
1,5 * \\
8,0^{*} \\
0,8 * \\
0,4^{*}\end{array}$ & $\begin{array}{l}3,6 \\
1,0 \\
0,2 \\
1,9 \\
0,1 \\
8,0 \\
1,4 \\
8,9 \\
0,9 \\
0,2\end{array}$ & $\begin{array}{l}0,7 \\
0,1 \\
0,1 \\
0,2 \\
0,1 \\
1,5 \\
0,9 \\
3,3 \\
0,1 \\
0,1\end{array}$ \\
\hline 30,5 & 25,8 & 22,9 & 24,9 & 21,8 & 21,6 & 25,6 & 26,1 & 7,1 \\
\hline $\begin{array}{r}41,5 \\
4,1\end{array}$ & $\begin{array}{r}38,1 \\
3,6\end{array}$ & $\begin{array}{r}31,1 \\
4,9\end{array}$ & $\begin{array}{r}7,6 \\
19,9\end{array}$ & $\begin{array}{r}6,0 \\
25,1\end{array}$ & $\begin{array}{r}6,8 \\
11,7\end{array}$ & $\begin{array}{r}10,5 \\
4,6\end{array}$ & $\begin{array}{r}13,0 \\
2,9\end{array}$ & $\begin{array}{l}2,2 \\
3,9\end{array}$ \\
\hline 76,1 & 67,5 & 58,9 & 52,4 & 52,9 & 40,1 & 40,7 & 42,0 & 13,2 \\
\hline
\end{tabular}

4. ' $\mathrm{H}$-Aktivität ausgeschieden (\%)

1. Urin

fester Rückstand

Kondensat

2. Kot

3. Atemluft

Summe $1-3$

$\begin{array}{r}15 \\ 0 \\ \hline 19\end{array}$

5. Bilanz

Mittelwert $=100,9$

* Schätzwerte aus Parallelversuchen

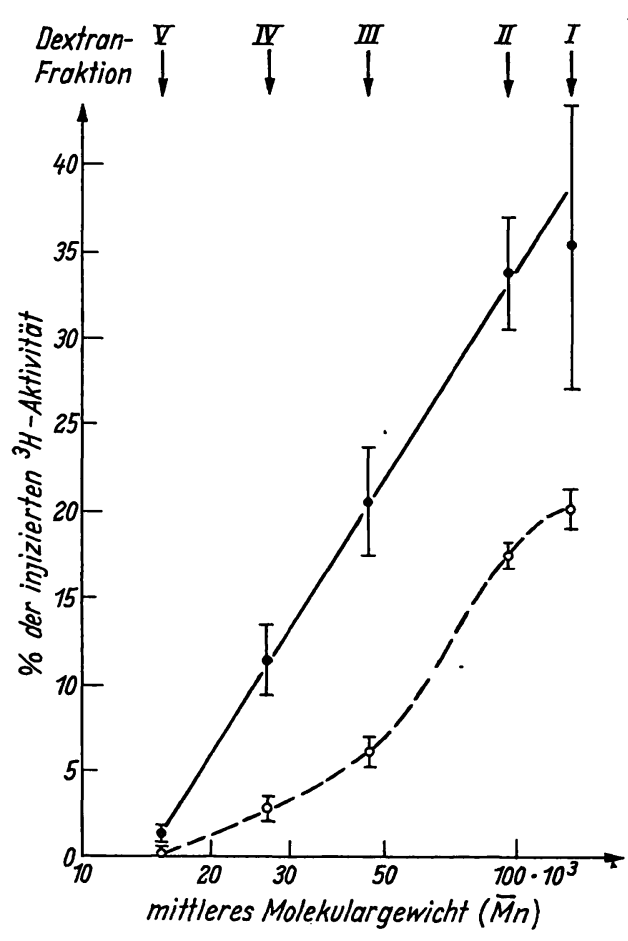

Abb. 2

Anteil der injizierten Tritium-Aktivitäten im Blut von MeerschweinAnteil der injizierten Tritium-Aktivitäten im Blut von Meerschweintritung teilung. Charakterisierung der Fraktionen siehe Tabelle 1 und Abbuldung Tritium-Wasser. Mittelwerte mit Standardabweichung von je 3-6 Tieren; insgesamt 40 Tiere

arität nur im nieder- und mittelmolekularen Bereich gegeben ist. Diese Dextrane sind nach 6 Tagen weit= gehend aus dem Blut verschwunden. Die stark verminderte Elimination der höhermolekularen Dextrane weist auf eine Beteiligung zusätzlicher Faktoren hin, die möglicherweise in einer "Nierenschwelle" für Dextrane zu suchen ist. Wie aus den Abbildungen 2 und $5 \mathrm{zu}$ erkennen ist, liegt diese Eliminationsschwelle bei einem Molekulargewicht um 50000. Dieser Befund stimmt überein mit den Beobachtungen anderer Autoren $(3,4)$, die eine Nierenșchwelle für Dextrane

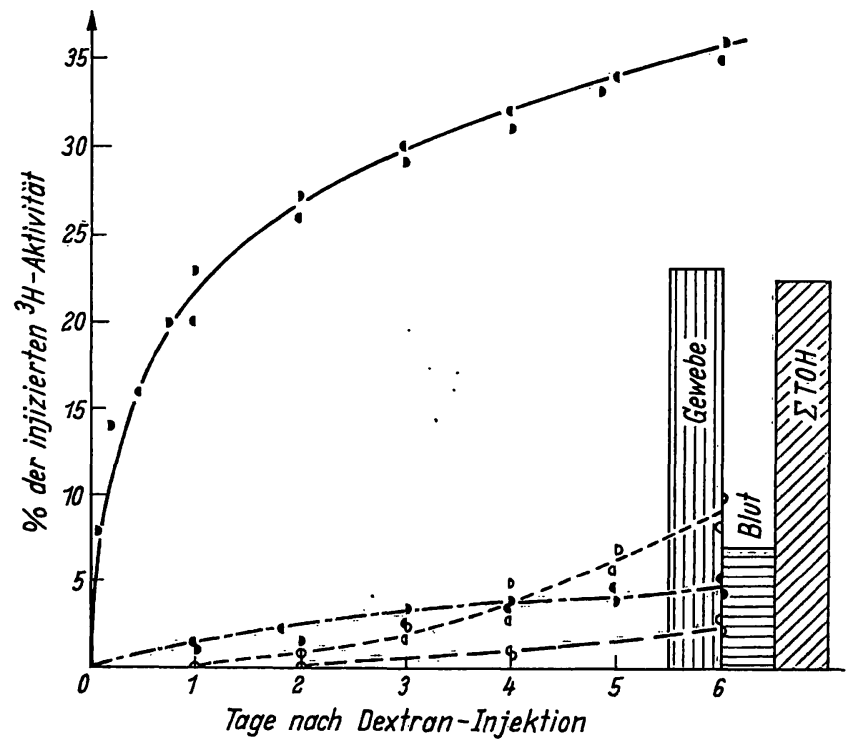

Abb. 3

Ausscheidung von Tritium-Aktivität im Urin-Rückstand $(-)$ und Kot (- - - ), sowie als Tritium-Wasser im Urin-Kondensat (- - ) und in der Ausatmungsluft (- $\rightarrow$ nach i. v. Injektion von Dextran Fraktion III (Tier $4 \rightarrow 0$; Tier 5 D; siehe Tab. 2).

Nachweis von Tritium-Aktivität nach sechstägiger Versuchsdauer im Gewebe und Blut (ohne TOH). Der Gehalt an Tritium-Wasser (TOH) wurde annäherungsweise berechnet aus der spezifischen Ak(TOH) wurde annäherungsweise berechnet aus der spezifischen Aktivität des ausgeatmeten Wassers und aus dem Lebendgewicht der
Tiere, unter der Annahme eines Wasseranteiles von $70 \%$ und einer Tiere, unter der Annahme eines Wasseranteiles von $70 \%$ und einer
gleichmäßigen Verteilung von TOH im gesamten Körperwasser. Mittelwert aus Tabelle 2 
bei eincm Molekulargewicht von etwa 55000 annehmen.

\section{Ausscheidung im Urin und Kot}

Wic die Abbildungen 3 und 4 sowie Tabelle 3 zeigen, wird der überwiegende Anteil der injizierten Dextrane über die Nieren und ein erheblich geringerer Anteil über den Darm ausgeschieden, wobei das Ausmaß der Aussrheidung dem Molekulargewicht umgekehrt proportional ist. Im Urin werden nach 3 Tagen mehr als $50 \%$ der niedermolekularen, $30 \%$ der mittel- und nur $15 \%$ der höhermolekularen Fraktionen wiedergefunden. Bei den ${ }^{3} \mathrm{H}$-Aktivitäten im Urin wurde zwischen dem festen Rückstand und dem Abkondensat unterschieden. ${ }^{3} \mathrm{H}$-Aktivitäten im Abkondensat - in Form von Tritium-Wasser - sind ein Indikator für den Dextranabbau und die Umsetzung im Stoffwechsel. Tritium-Wasser läßt sich in Spuren crst nach einem Tag, bei höhermolekularen Fraktionen sogar erst nach 3 Tagen nachweisen. Sein Anteil, bezogen auf die injizierten ${ }^{3} \mathrm{H}$-Aktivitäten, beträgt nach dreitägiger Versuchsdauer maximal 4\%. Die Hauptmenge der ${ }^{3} \mathrm{H}-$ Aktivität wird demnach im Urin ausgeschieden, bevor cin stärkcrcr Dextranabbau im Organismus cinsetzt. Die ${ }^{3} \mathrm{H}$-Aktivitäten, die im Urinrückstand gefunden wurden, müssen deshalb den injizicrten Dextranfraktionen entsprechen.

Im Kot lassen sich nur nach Injektion niedermolekularer Dextranfraktionen wesentliche ${ }^{3} \mathrm{H}$-Aktivitäten nachwcisen. Bei dicsen Fraktionen werden nach 3 Tagen über $10 \%$, bei mittel- und höhermolekularen Fraktionen dagegen weniger als $3 \%$ im Kot wiedergefunden, wobei die Halbwertszeit länger als bei der Ausscheidung über die Nieren ist. Bei Tier Nr. 6 (s. Tab. 3) findet man eine Anomalic: Der ${ }^{3} \mathrm{H}$-Aktivitätswert des Kots ist vergleichswcise sehr hoch, der

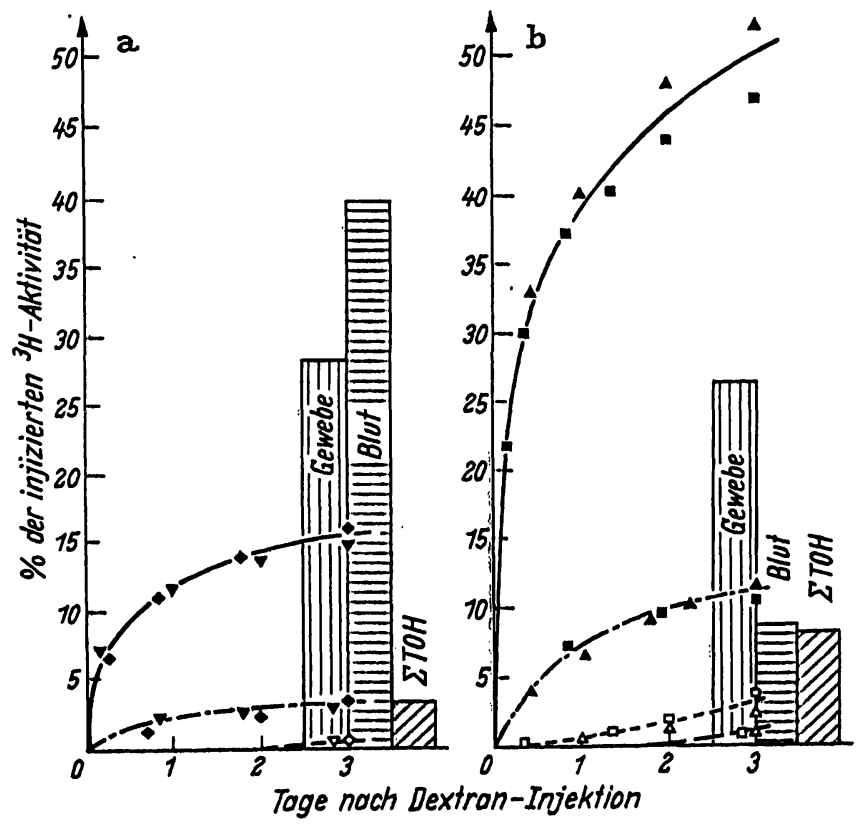

Abb. 4

Ausscheldung von Tritlum-Aktivitat in Urin, Kot und Ausatmungsluft und Nachwels in Korper, 3 Tage nach l. v. Injektion von a) Dextranfraktion II (Tier 7 2). Erkiurungen s. Legende zu Abbildung 3
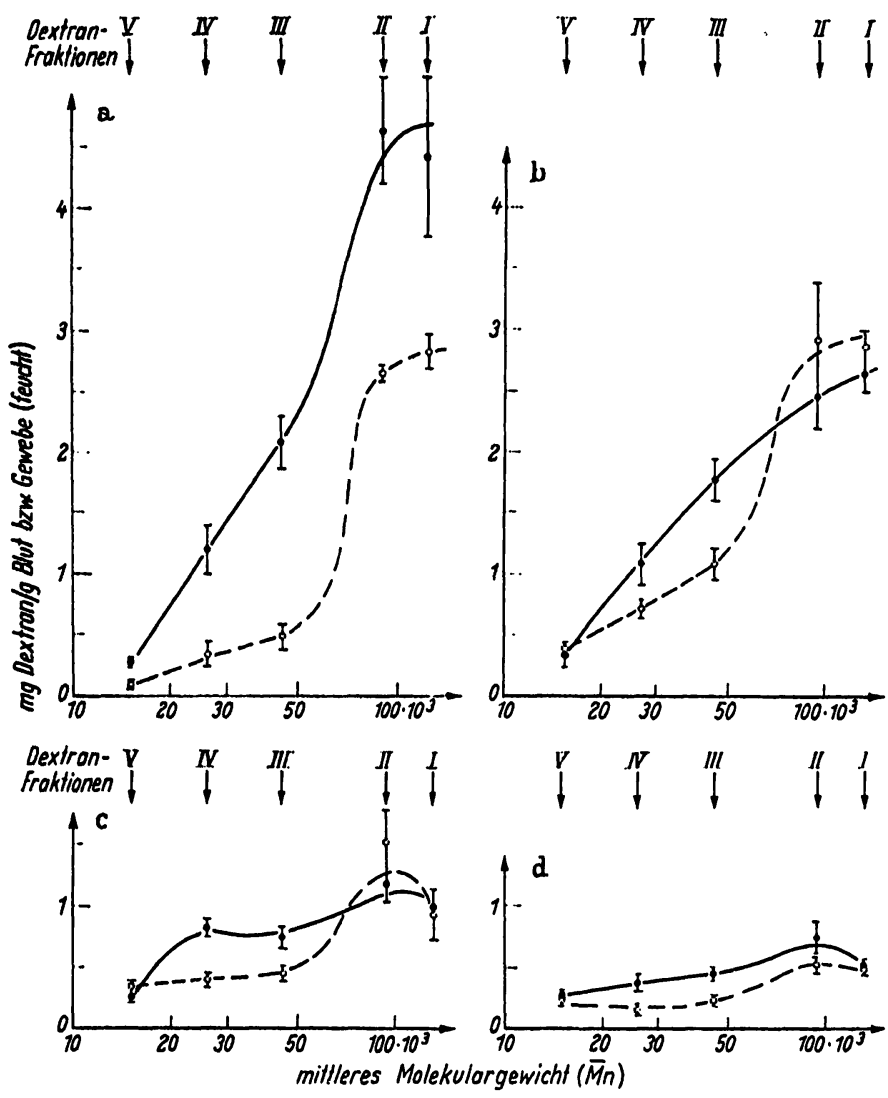

Abb. 5

Dextrankonzentrationen im Blut (a) und Dextrangehalt in Milz (b), Leber (c) und Nieren (d) von Mecrschwelnchen, 3 Tage $(\cdot-\bullet)$ und 6 Tage (o-.-o) nach i. v. Injektion von tritiierten Dextranen mit unterschiedlicher Molekulargewichtsvertellung (siehe Abb. 1), Die Dextranmengen wurden aus den gemessenen Aktivitäten in der Trockensubstanz von Blut und Geweben und aus der spezifischen Aktivltăt der einzelnen Dextranfraktionen (slehe Tab. 1) unter der Annahme berechnet, daß kein Tritium in körpereigene Substanzen
eingebaut wurde (siehe Text). Mittelwerte mit Standardabweichung en eingebaut wurde (siehe Text). Mittelwerte mit Standarcab
von je 3-6 Tieren; insgesamt 40 'Tiere

Wert des Urins dagegen sehr niedrig. Dicses Tier gab innerhalb der ersten 24 Std. im Stoffwechselkäfig keinen Urin $a b$.

\section{Metabolisierung}

Dextran kann durch enzymatische Hydrolyse zu Glucose abgebaut werden. In mehreren Säugetierorganen (12) und im Blut (18) wurden Dextranaseaktivitäten nachgewiesen. Dextran wird auf diesem Wege in den intermediären Stoffwechsel eingeschleust. Seine atomaren Bausteine müssen dann im $\mathrm{CO}_{2}$ der Atemluft, im Oxydationswasser und in Biosyntheseprodukten (z. B. Kohlenhydrate, Lipide, Proteine) erscheinen (7,9-11). In unseren Experimenten wurde cin tritiummarkiertes Dextran verwendet. Bei diescm Dextran ersetzt Tritium ausschließlich Wasserstoffatome, die an Kohlenstoff gebunden sind. Es war sichergestellt, daß keine in Wasser austauschbare C-OT-Aktivität im Dextran enthalten war. Tritium, das im Körperwasser, im Urinwasser oder im Wasscrdampf der Atemluft erscheint, muß deshalb von Dextran herrühren, das nach Zerlegung in seine Monomeren im encrgieliefernden Stoffwechsel (Glykolysc, Citratzyklus und Atmungskette) zu Kohlendioxid und Wasser verbrannt worden ist. Das Auftreten von Tritiumwasser ist deshalb ein sicherer Indikator für dic 
Metabolisierung des Dextrans. Wie oben bereits erwähnt wurde, hat das Auftreten von Tritiumwasser im Urin und in der Atemluft eine deutliche Induktionsperiode, die vom Molekulargewicht der Dextranfraktionen abhängig ist. Wie aus den Abbildungen 3 und $4 \mathrm{zu}$ erkennen ist, steigt die ${ }^{3} \mathrm{H}$-Aktivität im Urinwasser und in der Atemluft bei längerer Versuchsdauer zunehmend an. Dieser Effekt erfordert in quantitativer Hinsicht noch eine genauere Bearbeitung, insbesondere um zu prüfen, ob Anteile der H-OTAktivität aus Dextran auch außerhalb des Organismus der Versuchstiere gebildet werden, wofür wir allerdings keine Hinweise haben.

\section{Retention im Gewebe}

Tabelle 2 gibt eine Aufstellung der Organanalysen aus neun Einzelversuchen. Drei bzw. sechs Tage nach der Dextraninjektion lassen sich in sämtlichen untersuchten Geweben ${ }^{3} \mathrm{H}$-Aktivitäten nachweisen, die als Imp./Min./g Trockengewicht angegeben sind. Für weitere Umrechnungen enthält diese Tabelle die Angabe des Trockengewichtsanteils der Organfeuchtgewichte (Mittelwerte und Streuung). Die Analysen zeigen deutliche Unterschiede zwischen den einzelnen Organen. Die höchste spezifische Aktivität, bezogen auf das Organtrockengewicht, wird bei der Milz gefunden. Dann folgen Haut und Lunge und im weiteren Abstand Leber, Niere und Fettgewebe. Die Skelettmuskulatur enthält eine verhältnismäßig geringe spezifische Aktivität, die allerdings infolge der großen Muskelmasse einen erheblichen Anteil der injizierten Gesamt- ${ }^{3} \mathrm{H}$-Aktivität ausmacht. Im Gehirn sind nur Spuren an ${ }^{3} \mathrm{H}$-Aktivität nachweisbar, für die wahrscheinlich der Blutgehalt des Gewebes verantwortlich gemacht werden muß. (Diese Erklärung trifft sicherlich für die Analysen nach Injektion höhermolekularer Dextranfraktionen zu; sie erklärt jedoch nicht die Aktivitäten im Gehirn nach Injektion niedermolekularer Dextrane, nach der die ${ }^{3} \mathrm{H}$-Aktivität sehr rasch aus der Blutbahn verschwindet.) Die minimalen ${ }^{3} \mathrm{H}-$ Aktivitäten der Haare rühren sicher von Verunreinigungen her. Andererseits kann man so ausschließen, $\mathrm{da}$ die relativ hohen ${ }^{3} \mathrm{H}$-Aktivitäten der Haut durch äußerliche Verunreinigungen verursacht sind. Interessant ist der Befund einer höheren spezifischen Aktivität im intestinalen als im subkutanen Fettgewebe. Dieser Unterschied ist jedoch nur nach Injektion höhermolekularer Fraktionen deutlich ausgeprägt.

Die Retention von ${ }^{3} \mathrm{H}$-Aktivitäten in Milz, Leber und Nieren wurde in einer größeren Versuchsserie untersucht und der Retention im Blut gegenübergestellt. In Abbildung 5 sind die spezifischen Aktivitäten auf das Dextrangewicht umgerechnet, das diesen Aktivitäten entspricht, sofern kein Dextran abgebaut wurde. Die Dextrangehalte wurden auf das Feuchtgewicht der Organe bezogen, um einen Vergleich mit den Dextrankonzentrationen im Blut zu ermöglichen.

Wie ein Vergleich mit den Dextrankonzentrationen im Blut zeigt, kann der Dextrangehalt dieser Organe nicht mit ihrem Blutgehalt erklärt werden. Es findet also bei allen Organen eine Dextrananreicherung im Gewebe statt, die bei der Milz mehrfach stärker ist als bei der Leber und den Nieren. Die Dextranretention ist molekulargewichtsabhängig. Die Abhängigkeit ist bei der Milz besonders ausgeprägt. Die Dextranretention spiegelt nicht die Dextrankonzentration im Blut wider; sie verhält sich in der Milz teilweise sogar entgegengesetzt. So nimmt nach Injektion höhermolekularer Fraktionen der Dextrangehalt der Milz bei längerer Versuchsdauer zu, während die Dextrankonzentration im Blut abnimmt. Der hohe Dextrangehalt in der Milz und auch der gegenüber den Nieren relativ hohe Gehalt in der Leber weist darauf hin, daß dem reticuloendothelialen System eine bedeutende Rolle bei der Resorption hochmolekularer Dextrane, deren Ausscheidung über Darm und Nieren erschwert ist, zukommt. Es muß jedoch betont werden, daß die ${ }^{3} \mathrm{H}$-Aktivitäten, die in den gefriergetrockneten Geweben gemessen wurden, nicht ausschließlich Dextran entsprechen müssen. Wie bereits oben ausgeführt, wird Dextran nach einer Induktionsperiode in den Stoffwechsel eingeschleust. ${ }^{3} \mathrm{H}$-Aktivitäten in der Trockensubstanz der Gewebe können daher auch körpereigenen Verbindungen entsprechen. Das trifft mit größerer Wahrscheinlichkeit zu für Analysen aus stoffwechselaktiven Organen, wie z. B. Leber und Fettgewebe, und nach längerer Versuchsdauer.

Andererseits scheint der Anteil der retinierten ${ }^{3} \mathrm{H}-$ Aktivität, der nicht dem Dextran zugeordnet werden kann, jedoch unerheblich zu sein. Bei Tieren, die 4 Tage nach Dextraninjektion und nach 24stdg. Nahrungsentzug getötet wurden, konnte im TritiumGehalt des Lebergewebes kein meßbarer Unterschied gegenüber normal ernährten Tieren festgestellt werden. Leberglykogen, das bei den hungernden Tieren zu mehr als $90 \%$ abgebaut war, konnte somit keine wesentliche Menge radioaktiver Glucoseeinheiten enthalten haben.

\section{Bilanzen}

Die Bilanzen der Stoffwechselversuche aus Tabelle 2 sowie aus den Abbildungen 3 und 4 sind in Tabelle 3 zusammengefaßt. Diese Tabelle enthält in Abschnitt 1 und 2 das Tiergewicht, die Versuchsdauer und Angaben über die injizierte Dextranlösung. In Abschnitt 3 sind die ${ }^{3} \mathrm{H}$-Aktivitäten in den wichtigsten Organen als Prozent der injizierten Gesamtmenge angegeben. Der Berechnung liegen die spezifischen Aktivitäten aus Tabelle 2 und die Organgewichte zugrunde, soweit letztere erfaßt werden konnten. Fettgewebe konnte begreiflicherweise nur unvollständig gesammelt werden. Bei mehreren Versuchstieren wurden nicht alle Organe untersucht. Hier wurden Werte aus Parallelversuchen eingesetzt, die besonders gekennzeichnet sind. Für Blut und Körperwasser wurden als Schätzwerte $10 \%$ bzw. $70 \%$ des Lebendgewichtes vòr der Tötung angenommen. Alle Angaben, mit Ausnahme des Körperwassers, beziehen sich auf die ${ }^{3} \mathrm{H}$-Aktivität im gefrier- 
getrockneten Gewebe bzw. im Blutrückstand. Sie enthalten folglich kein Tritiumwasser. Eine Analyse des Blutkondensats wurde nicht vorgenommen. Statt dessen wurde die ${ }^{3} \mathrm{H}$-Aktivität im Körperwasser aus der spezifischen Aktivität im kondensierten Wasser der Atemluft berechnet. Die Berechnung geht von folgenden Annahmen aus: 1. Das Tritiumwasser in der Abluft des Käfigs stammt aus dem intermediären Stoffwechsel des Meerschweinchens. 2. Die spezifische Aktivität des Wassers, das im trockenen Käfig in den letzten Stunden vor der Tötung des Tieres entsteht, entspricht der spezifischen Aktivität des Körperwassers.

Der Anteil der injizierten Aktivität, der 3 Tage nach der Dextraninjektion im Körper wiedergefunden wurde, liegt zwischen $76 \%$ bei der höhermolekularen und $13 \%$ bei der niedermolekularen Fraktion. Diese hohe Abhängigkeit vom Molekulargewicht des injizierten Dextrans wird allerdings vorwiegend durch die Verweildauer im Blut bestimmt (s. Abb. 2), während die Gesamt- ${ }^{3} \mathrm{H}$-Aktivität der in den Geweben nachgewiesenen ${ }^{3} \mathrm{H}$-Aktivitäten mit Ausnahme der kleinstmolekularen Fraktion (Fraktion V) sich weitgehend angleicht. Die molekulargewichtsabhängige Retention in einzelnen Organen, z. B. in der Milz (s. Abb. 5) ist für die Bilanz ohne Bedeutung. Abschnitt 4 der Tabelle 3 bringt den Anteil der injizierten ${ }^{3} \mathrm{H}$-Aktivitäten, der im Urin, im Kot und in der Atemluft wiedergefunden wurde. Der Ablauf dieser Ausscheidungen wurde in den Abbildungen 3 und 4 beschrieben und bereits oben diskutiert. Innerhalb von 3 Tagen nach der Dextraninjektion wurden zwischen $20 \%$ und $86 \%$ ausgeschieden. Diese Werte entsprechen im umgekehrten Sinne den Blutwerten, so daß die Bilanzen der einzelnen Versuche nahe $100 \%$ liegen. Nach den vorliegenden Ergebnissen ist die molekulargewichtsabhängige Verweildauer von Dextran im Blut deshalb vorwiegend eine Funktion der Ausscheidung. Retention oder Metabolisierung in den Geweben sind in dieser Hinsicht von untergeordneter Bedeutung.

Wir danken der Knoll AG, Chemische Fabriken, Lud wigshafen a. Rh., für die großzügige Unterstützung dieser Arbeiten, Frau Dr. K. Granath, Pharmacia AB, Uppsala, für die Ausführung der Molekulargewichtsverteilungsmessungen, Herrn Dr. J. KLEIN für die Durchführung der präparativen Fraktionierung und Herrn Mechanikermeister AlbERT SCHALlweg für Konstruktion und Anfertigung des Stoffwechselkäfigs.

\title{
Literatur
}

1. Grotte, G., R. C. Knutson und J. L. Bollmann, J. Laborat. Clin. Med. S. Louis 38, 577 (1951). - 2. Arturson, G. und G. Wallenius, Scand. J. Clin. Laborat. Inivest. 1, 76 (1964). - 3. Arturson, G. und G. Wallenius, Scand. J. Clin. Laborat. Invest. 1, 81 (1964). - 4. Arturson, G., K. Granath und G. Grotre, Arch. Dis. Childh. London 41, 168 (1966). - 5. SchwartzkopfF, W., Verh. Dtsch. Ges. inn. Med. 70, 705 (1964). 6. Arturson, G. Scand. J. Clin. Laborat. Invest. 17, Suppl. 86, 69 (1965). - 7. Hellmann, Prel. Report on the Metabolism of C-14 Labelled Dextran and PVC, Report to National Research Council, October 1951. - 8. Aberg, B., W. L. Bloom und E. Hansson, Acta physiol. Scand. 52, 188 (1961). - 9. Terry, R., Ch. L. Yuile; A. Golodetz, C. E. Phillips und R. R. Whrte, J.
Laborat. Clin. Med. S. Louis 42, 6 (1953). 10. Gray, I. P. K. Sitery und E. J. Pulaski, Proc. Soc. exper. Biol Med. 77, 626 (1951). - 11. Gray, I., Amer. J. Physiol. 174 462 (1953). - 12. Rosenfeld, E. L. und A. S. Satenko, Clin. chimica Acta Amsterdam 10, 223 (1964) - - 13. Ebert, K. H. und M. Brosche, Biopolymers 5, 423 (1967). - 14. EbERT, K. H., G. Rupprecht und G. Schenk, Z. Naturforsch. 18b, 442 (1963). 15. Granath, K. A. und B. E. Kvist, J. Chromatog. 28, 69 (1967). - 16. Klein, J. und E. KillmanN, Makromol. Chem. 96, 193 (1966). - 17. Kalberer, F. und J. Rutschmann, Helv. chim. Acta 44, 1957 (1961). - 18. ChytiL, F, L. Lacko und O. STĚRBA, Blut XII, 310 (1966).

Doz. Dr. K. Ebert 8000 München 2 Arcisstr. 21

\section{A New Method for the Determination of Serum Nucleotidase}

\author{
By J.-P. Persijn, W. van Der Stik, K. KRAmer and C. A. De Ruijter \\ From the Department of Clinical Chemistry (Head, Dr. J.-P. Persijn), Netherlands Cancer Institute, Amsterdam, and the \\ Department of Clinical Chemistry, State University Hospital, (Head, Drs. W. van der Slik), Leiden, The Netherlands.
}

(Eingegangen am 8. Mai 1968)

A new method for the determination of serum nucleotidase (EC 3.1.3.5) is described; its accuray and reproducibility have been investigated. For determinations in serum the coefficients of variation were $2.5-3.5 \%$.

The serum $5 \mathrm{~N}$ activity is measured by the amount of $\mathrm{NH}_{3}$ liberated from adenosine after incubation with the enzyme adenosine deaminase (EC 3.5.4.2).

Eine neue Methode für die Bestimmung der Serum-Nucleotidase (EC 3.1.3.5) wird beschrieben, ihre Genauigkeit und Reproduzierbarkeit untersucht. Für die Bestimmung im Serum betragen die Variationskoeffizienten 2,5-3,5\%.

Die Aktivität des Serums gegenüber AMP wird nach Inkubation mit Adenosindesaminase (EC 3.5.4.2) bestimmt, gemessen wird das dabei freigesetzte Ammoniak. 